\title{
Demonstration of Launch Vehicle Slosh Instability on Pole-Cart Platform
}

\author{
Jing Pei* and Paul Rothhaar ${ }^{\dagger}$ \\ NASA Langley Research Center, Hampton, Virginia, 23681
}

\begin{abstract}
Liquid propellant makes up a significant portion of the total weight for large launch vehicles such as Saturn V, Space Shuttle, and the Space Launch System (SLS). Careful attention must be given to the influence of fuel slosh motion on the stability of the vehicle. A well-documented slosh danger zone occurs when the slosh mass is between the vehicle center of mass and the center of percussion. Passive damping via slosh baffle is generally required when the slosh mass is within this region. The pole-cart hardware system, typically used for academic purposes, has similar dynamic characteristics as an unstable launch vehicle. This setup offers a simple and inexpensive way of analyzing slosh dynamics and its impact on flight control design. In this paper, experimental and numerical results from the pole-cart system will be shown and direct analogies to launch vehicle slosh dynamics will be made.
\end{abstract}

\section{Introduction}

Liquid propellant makes up a significant portion, as much as 90 percent during liftoff ${ }^{1}$ of the launch vehicle gross weight. The effect of fuel sloshing on vehicle dynamics and control must be taken into consideration in the design of the flight control system. This is especially true for slosh tanks with large diameters in which the frequencies of the slosh masses approach the rigid body control frequency. A poorly designed flight control system can cause continuous excitation of the slosh dynamics, hence making the closed-loop system unstable. In 2007, the SpaceX Falcon demonstration flight II exhibited a noticeable slow divergent oscillation in the roll channel upper stage control system. The instability grew and manifested itself in pitch and yaw until second stage engine shutdown. The instability prevented the vehicle from reaching its intended orbit. Subsequent post flight investigation ${ }^{2}$ described this phenomenon as a partial loss of control and pointed out that the Falcon 1 did not use slosh baffles, in the second stage tanks as slosh instability was deemed to be a low risk at the time.

Typically in the formulation of the linearized equations of motion for a launch vehicle, ${ }^{3,4}$ the slosh dynamics is modeled as either a spring-mass or a pendulum. In both cases, the displacement of the slosh mass is assumed to be small. There are several key slosh parameters that affect the vehicle stability: 1) Magnitude of the slosh mass or portion of the total liquid mass is assumed to behave as an equivalent pendulum. The inertia ratio metric is used to quantify the impact of a particular slosh tank on control stability. The inertia ratio is the ratio of the transverse moment of inertia of the slosh mass to that of the integrated vehicle. 2) Frequency of the slosh mode, which is dependent on the length and the mass of the equivalent pendulum representation and the vehicle axial acceleration. 3) Location of the slosh mass along the length of the vehicle.

Bauer $^{5}$ and Greensite ${ }^{3}$ mathematically demonstrated using a simple vehicle dynamics model (rigid body rotation and slosh) that the danger zone for slosh instability occurs when the slosh mass $\left(M_{p}\right)$ is between the vehicle center of mass $(\mathrm{CM})$ and center of percussion $(\mathrm{CP})$ or instantaneous center of rotation. Center of percussion ${ }^{6}$ is defined as a point on the body where the translational motion perfectly cancels out the rotational motion at a specific instant in time. Center of percussion for a rocket can be determined by: ${ }^{3}$

$$
C P=\frac{I_{y y}}{L_{c} M}
$$

*Aerospace Engineer, Systems Analysis and Concepts Directorate, Vehichle Analysis Branch

${ }^{\dagger}$ Aerospac Engineer, Research Directorate, Dynamics and Control Branch 
Here CP is defined as a point on the vehicle foward the mass center. $I_{y y}$ is the transverse moment of inertia of the rocket with respect to the mass center, $L_{c}$ is the distance from the thrust application point to the $\mathrm{CM}$, and $M$ is the total mass of the vehicle.

Once inside the danger zone, the slosh mass has unfavorable phasing with respect to rigid body control or phase unstable. In other words, the effector is indirectly adding energy into the slosh mode when trying to actively control the rigid body attitude. Figure 1 highlights the difference between a phase stable vs. phase unstable slosh mode on a root locus plot (zoomed in on the slosh frequency). In the stable case, the slosh pole is above the zero and the angle of departure associated with the pole is into the left half plane, hence the closed-loop slosh pole is stable for all values of feedback gains. For the unstable case, the slosh pole is below the zero, the angle of departure for the unstable slosh mode is into the right half plane and the system is stable for only a narrow band of loop gain (assume negligible damping). As a result, additional damping via baffles is required to ensure the closed-loop slosh pole remains stable. Figure 2 illustrates the difference on a Nichols chart. The frequency response associated with the stable slosh mode naturally projects away from the critical point, whereas for unstable slosh the frequency response protrudes towards the critical point. If damping does not exist then encirclement of the critical point would occur.
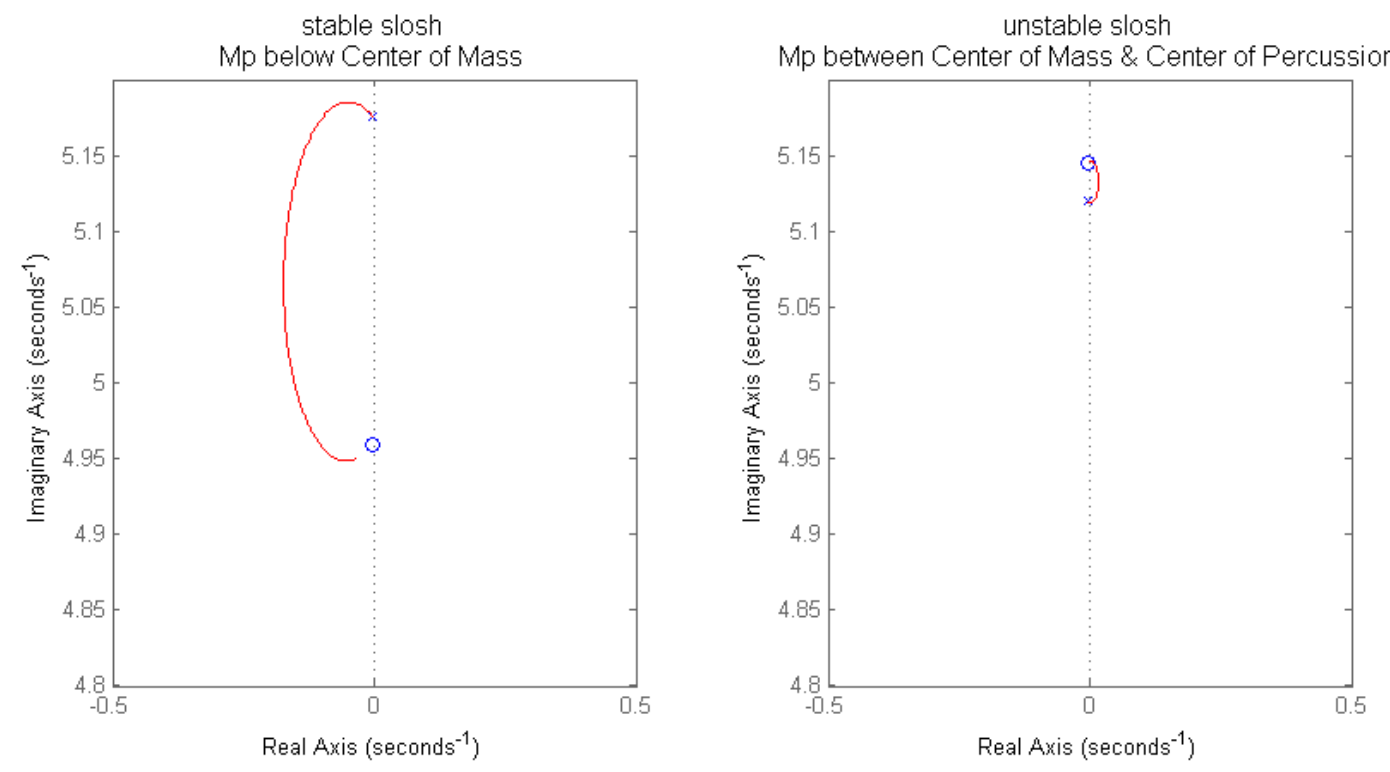

Figure 1. Phase Stable vs. Unstable Slosh: Root Locus

Figures 3 and 4 show results from a time domain simulation of a thrust vector controlled rocket with a slosh mass that is located outside/inside the danger zone. The system is given an initial disturbance and the control system attempts to regulate the vehicle attitude. Figure 3 is a phase portrait of the slosh angle and velocity when the slosh is outside the danger zone, hence it is phase stable. Figure 4 illustrates what happens when the slosh mass is inside the danger zone. The slosh angle slowly diverges due to unstable closed-loop slosh pole. Time-to-double is generally large compare to rigid body instability.

There are two major liquid propellant tanks located on the SLS vehicle: LH2 (hydrogen) and LOX (oxygen). Other than for the first 10 seconds, the LH2 slosh (in red) is phase stable throughout the entire ascent flight. The LOX slosh (in green) starts out phase stable and becomes phase unstable during the core phase of flight, after the solid rocket boosters have separated. Additional baffles are required to passively stabilize the LOX mode. Figure 5 shows the locations of the slosh masses along with the vehicle CM (blue) and $\mathrm{CP}$ (cyan) time histories. Bauer ${ }^{5}$ also drew four important conclusions of a rigid vehicle with one propellant slosh tank: 1) increasing slosh mass decreases stability, 2) decreasing the slosh natural frequency shifts the danger zone towards the aft of the vehicle and requires more damping for stability, 3) increasing the slosh natural frequency diminishes the impact of slosh on rigid body stability, 4) a decrease in the attitude control system gain shifts the danger zone towards the aft of the vehicle.

The inverted pendulum on a cart (pole-cart) is a classical system widely studied in academia ${ }^{7-10}$ and control theory. Similar to a rocket, the inverted pendulum (IP) is open-loop unstable. In both cases, feedback 


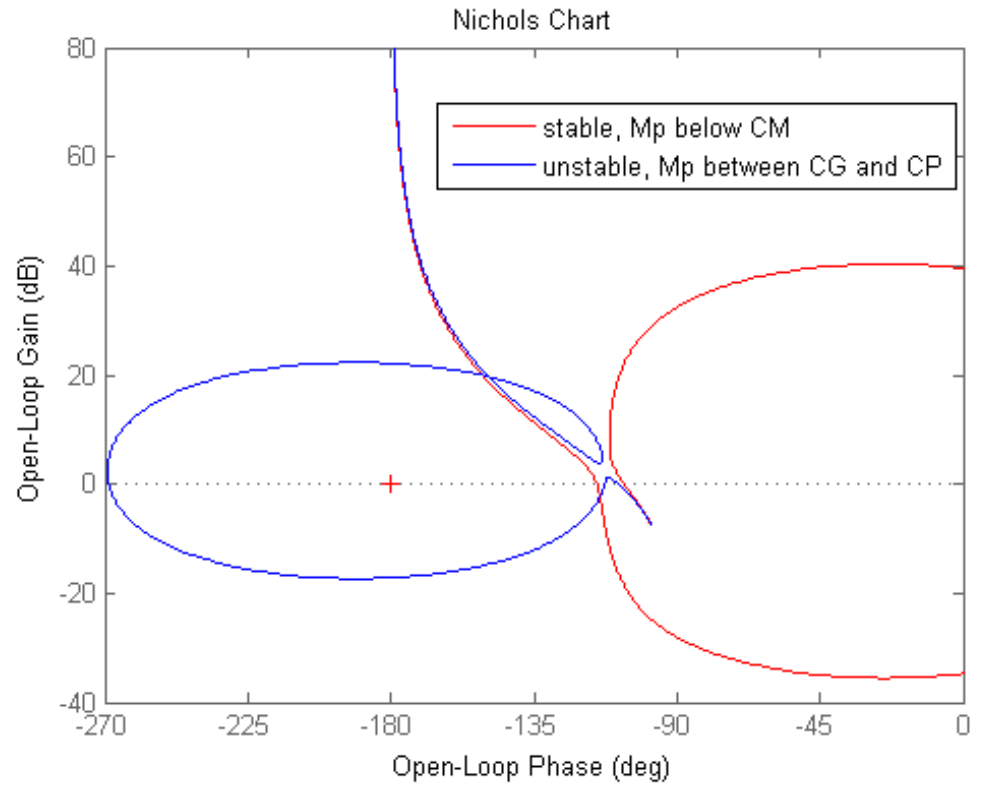

Figure 2. Phase Stable vs. Unstable Slosh: Nichols Chart

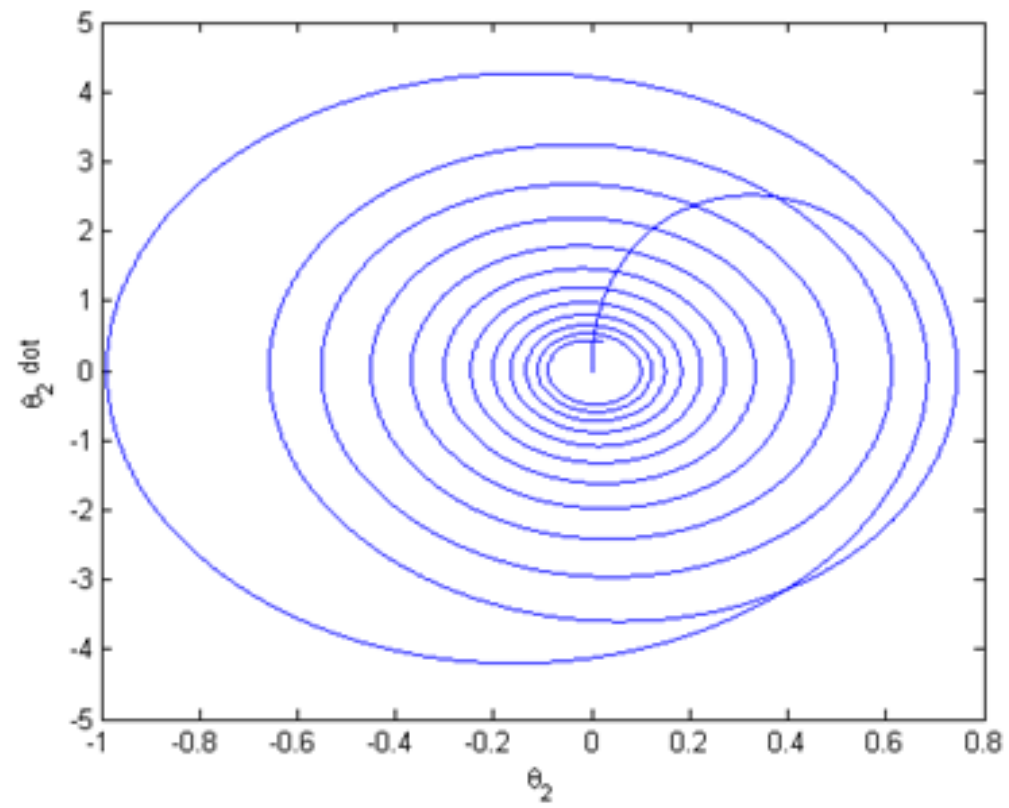

Figure 3. Phase Portrait of Stable Slosh 


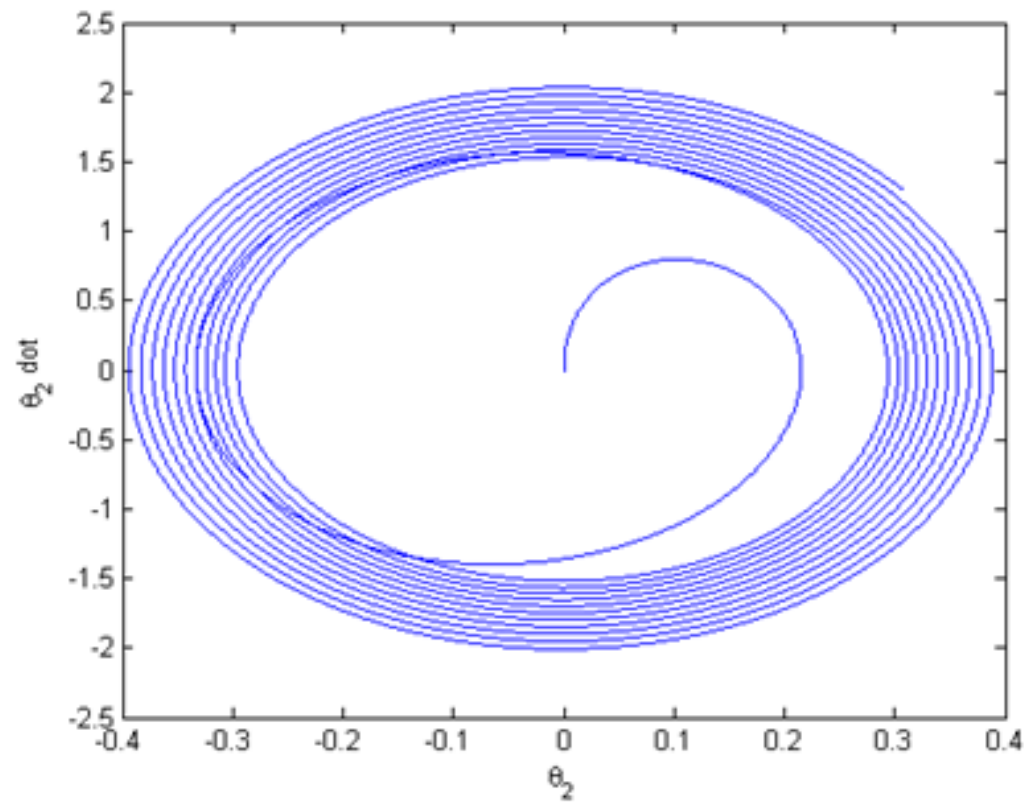

Figure 4. Phase Portrait of Unstable Slosh
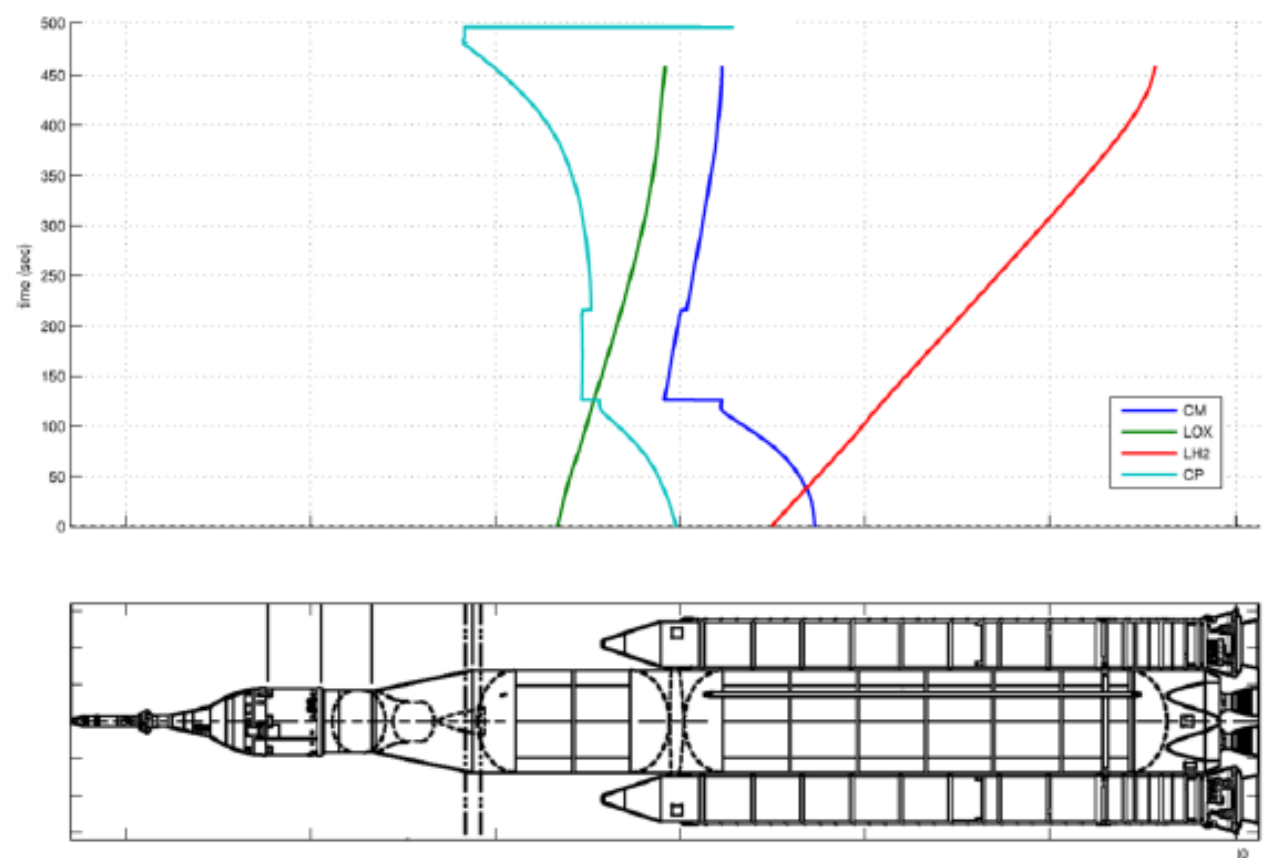

Figure 5. SLS Slosh Tank Locations Time History 
control is required to maintain system stability. The effect of slosh parasitic dynamics can be modeled by attaching a secondary pendulum to the IP. This paper is describes how the pole-cart hardware can used to demonstrate the effect of slosh on the rigid body control of a typical launch vehicle. The remainder of this paper is organized as follows: Section II derives the equations of motion (nonlinear and linear) for the generic double pendulum-cart system and provides an analogy between that and the coupled dynamics of a slosh mass with a rocket. Section III discuss the experimental setup. Section IV shows results from the experiment and numerical simulations, where additional discussions are made on how the pole-cart results relate to slosh on a launch vehicle. Section V summarizes the results and provides discussion for future work.

\section{Pole-Cart Dynamics}

The pole-cart system is a classical setup used in academia to illustrate principles of controls engineering. The objective is to move the cart back and forth on the track to actively stabilize the inverted pendulum. The inverted pendulum (IP) is an inherently an unstable system and can be used to represent the planar dyamics of a typical aerodynamically unstable launch vehicle. Both systems have a real pole on the right half of the s-plane and require active feedback control for stabilization. The cart is analogous to the control effector(s) that are located at the base of a launch vehicle. To mimic the effect of fuel sloshing, a second or slosh pendulum (SP) is attached to the IP. Figure 6 is an illustration showing the similarities between the two systems. The key difference being the launch vehicle is free to rotate about its center of mass, whereas the IP rotates about the pivot point at which it is attached to the cart.

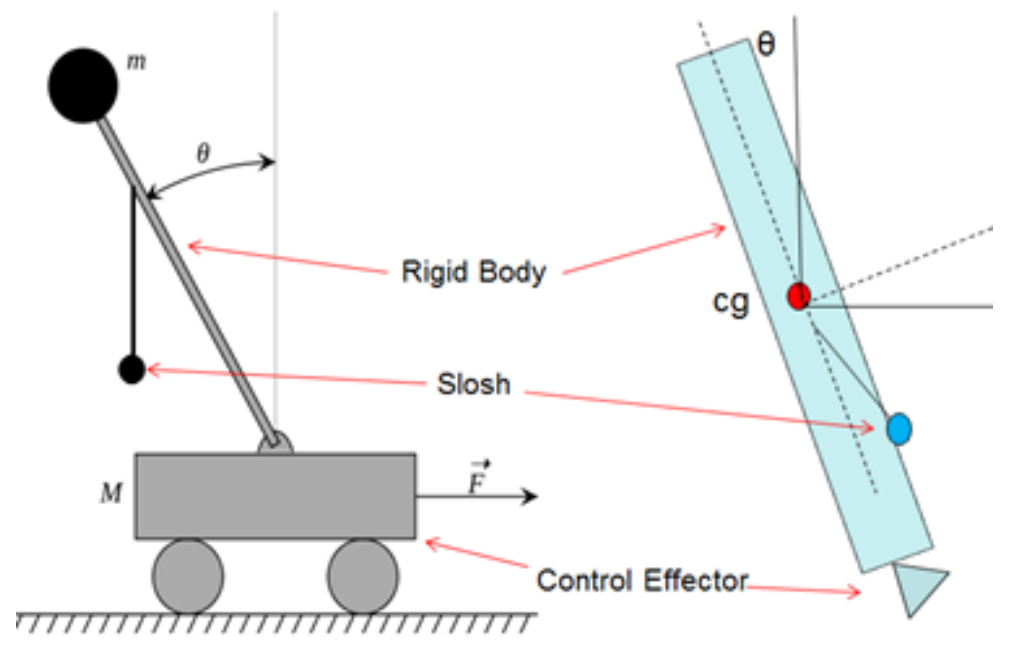

Figure 6. Dynamic Similarity Between Pole-Cart and Launch Vehicle

Lagrange's method ${ }^{11}$ was used to derive the equations of motion of the cart and double pendulum configuration. Figure 7 shows the nomenclature and sign conventions that were adopted in the derivation. $m_{c}, m_{1}, m_{2}$ are the mass of the cart, IP, and SP respectively. $l_{1}$ and $l_{2}$ are the total length of the pendulums. $J_{1}$ and $J_{2}$ are the inertias of the pendulums about the respective center of mass. $L$ is the location along the IP at which the SP will be attached (distance between the pivot points P and Q). Note: the axes and angular definitions can be a source of great confusion when comparing different pendulum EOM derivations. Equations 3 through 8 illustrates the nonlinear system of equations and follow the assumption that the mass is distributed uniformly in each link of the pendulum so that the mass centers are located at $l_{1} / 2$ from $\mathrm{P}$ and $l_{2} / 2$ from $\mathrm{Q}$ respectively. 


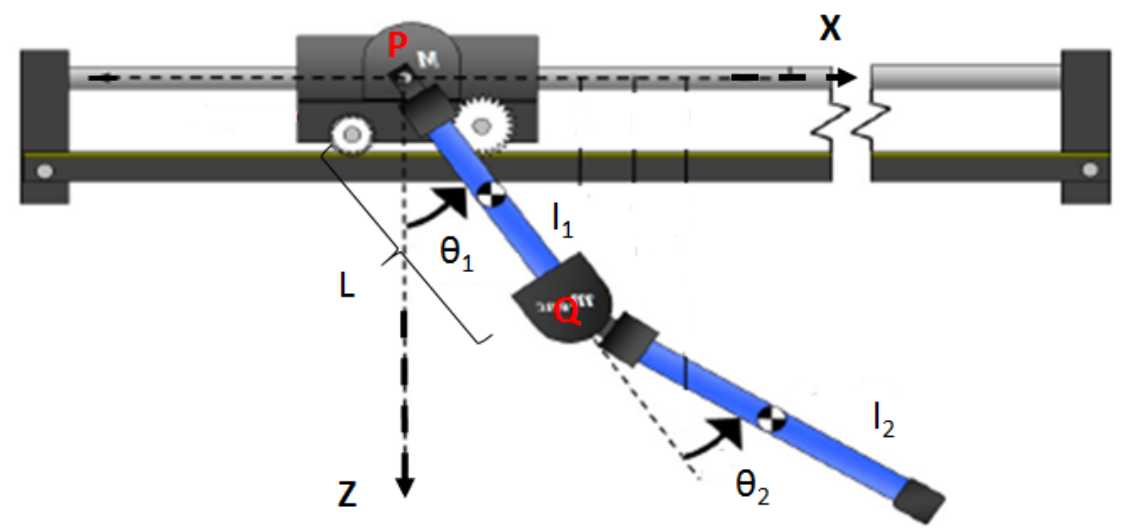

Figure 7. Nomenclature and Sign Conventions

Using the angular orientation, axes, and lengths from Fig. 7. The Lagrangian can be expressed as the total kinetic $(T)$ and potential $(V)$ energy of the system shown in Eq. (2) and the generlized coordinates of the system are: $x, \theta_{1}, \theta_{2}$.

$$
\begin{aligned}
L=T-V & =\frac{1}{2}\left(m_{1}+m_{2}+m_{c}\right) \dot{x}^{2}+\left(\frac{1}{8} m_{1} l_{1}^{2}+\frac{1}{2} m_{2} L^{2}+\frac{1}{8} m_{2} l_{2}^{2}\right) \dot{\theta}_{1}^{2}+\frac{1}{8} m_{2} l_{2}^{2}{\dot{\theta_{2}}}^{2} \\
& +\frac{1}{4} m_{2} l_{2}^{2} \dot{\theta_{1}} \dot{\theta_{2}}+\left(\frac{1}{2} m_{1} l_{1}+m_{2} L\right) \dot{x} \dot{\theta_{1}} \cos \theta_{1}+\frac{1}{2} m_{2} l_{2} \dot{x} \cos \left(\theta_{1}+\theta_{2}\right)\left(\dot{\theta_{1}}+\dot{\theta_{2}}\right) \\
& +\frac{1}{2} m_{2} L l_{2} \dot{\theta_{1}}\left(\dot{\theta_{1}}+\dot{\theta_{2}}\right) \cos \theta_{2}+\frac{1}{2}{J_{1}}_{\dot{\theta}_{1}}{ }^{2}+\frac{1}{2} J_{2}\left(\dot{\theta_{1}}+\dot{\theta_{2}}\right)^{2}+\frac{1}{2} m_{1} g l_{1} \cos \theta_{1} \\
& +m_{2} L g \cos \theta_{1}+\frac{1}{2} m_{2} l_{2} g \cos \left(\theta_{1}+\theta_{2}\right)
\end{aligned}
$$

Following Lagrange's approach, the final nonlinear equations of motion can be shown in a matrix representation shown in Eq. (3). The states are the generalized coordinates and their time derivatives.

$$
\left[\begin{array}{c}
\ddot{x} \\
\ddot{\theta}_{1} \\
\ddot{\theta}_{2}
\end{array}\right]=\left[\begin{array}{lll}
a_{11} & a_{12} & a_{13} \\
a_{21} & a_{22} & a_{23} \\
a_{31} & a_{32} & a_{33}
\end{array}\right]^{-1}\left[\begin{array}{c}
b_{1} \\
b_{2} \\
b_{3}
\end{array}\right]
$$

where $\mathrm{A}$ is the matrix with elements aij which are:

$$
\begin{aligned}
& a_{11}=m_{1}+m_{2}+m_{c} \\
& \left.a_{12}=\frac{1}{2} m_{1} l_{1}+m_{2} L\right) \cos \theta_{1}+\frac{1}{2} m_{2} l_{2} \cos \left(\theta_{1}+\theta_{2}\right) \\
& a_{13}=\frac{1}{2} m_{2} l_{2} \cos \left(\theta_{1}+\theta_{2}\right) \\
& a_{21}=\left(\frac{1}{2} m_{1} l_{1}+m_{2} L\right) \cos \theta_{1}+\frac{1}{2} m_{2} l_{2} \cos \left(\theta_{1}+\theta_{2}\right) \\
& a_{22}=\frac{1}{4} m_{1} l_{1}^{2}+m_{2} L^{2}+\frac{1}{4} m_{2} l_{2}^{2}+m_{2} L l_{2} \cos \theta_{2}+J_{1}+J_{2} \\
& a_{23}=\frac{1}{4} m_{2} l_{2}^{2}+\frac{1}{2} m_{2} L l_{2} \cos \theta_{2}+J_{2} \\
& a_{31}=\frac{1}{2} m_{2} l_{2} \cos \left(\theta_{1}+\theta_{2}\right) \\
& a_{32}=\frac{1}{4} m_{2} l_{2}^{2}+\frac{1}{2} m_{2} L l_{2} \cos \theta_{2}+J_{2} \\
& a_{33}=\frac{1}{4} m_{2} l_{2}^{2}+J_{2}
\end{aligned}
$$

and the terms $b_{1}, b_{2}$, and $b_{3}$ are 


$$
\begin{aligned}
& b_{1}=F+\left(\frac{1}{2} m_{1} l_{1}+m_{2} L\right) \sin \theta_{1}{\dot{\theta_{1}}}^{2}+\frac{1}{2} m_{2} l_{2} \sin \left(\theta_{1}+\theta_{2}\right)\left(\dot{\theta}_{1}+\dot{\theta}_{2}\right)^{2} \\
& b_{2}=\tau_{1}+m_{2} L l_{2}\left(\dot{\theta}_{1}+\frac{1}{2} \dot{\theta}_{2}\right) \sin \theta_{2} \dot{\theta}_{2}-\left(\frac{1}{2} m_{1} l_{1}+m_{2} L\right) g \sin \theta_{1}-\frac{1}{2} m_{2} l_{2} g \sin \left(\theta_{1}+\theta_{2}\right) \\
& b_{3}=\tau_{2}-\frac{1}{2} m_{2} L l_{2} \dot{\theta}_{1}^{2} \sin \theta_{2}-\frac{1}{2} m_{2} l_{2} g \sin \left(\theta_{1}+\theta_{2}\right)
\end{aligned}
$$

The forces and torques include both the motor force $\left(f_{\text {motor }}\right)$ and system friction coefficients $\left(k_{\dot{x}} \dot{x}, k_{\dot{\theta}_{1}}\right.$, $\left.k_{\dot{\theta}_{2}}\right)$ :

$$
\begin{aligned}
F & =k_{\dot{x}} \dot{x}+f_{\text {motor }} \\
\tau_{1} & =k_{\dot{\theta}_{1}} \dot{\theta}_{1} \\
\tau_{2} & =k_{\dot{\theta}_{2}} \dot{\theta}_{2}
\end{aligned}
$$

The inverse of the non-linear state dependent mass matrix comprised of elements $a_{i j}$ is computed at each integration interval for simulation. In order to mimic the short period dynamics of a launch vehicle, the nonlinear cart double-pendulum EOM were linearized about the desired operating condition: $x=0, \theta_{1}=\pi$, $\theta_{2}=\pi, \dot{x}=0, \dot{\theta}_{1}=0, \dot{\theta}_{2}=0$ and put into the standard state space $\dot{x}=A x+B u$ format. Equations 9 through 12 are elements of the linearized A and B matrices without motor dynamics.

$$
A=\left[\begin{array}{cc}
0_{3 \times 3} & I_{3 \times 3} \\
-H^{-1} \frac{\partial G}{\partial q} & 0_{3 \times 3}
\end{array}\right]
$$

where $\mathrm{H}$ is the inverse of the linearized $\mathrm{A}$ matrix and $\frac{\partial G}{\partial q}$ are the stiffness terms dependent on $\theta_{1}$ and $\theta_{2}$

$$
\begin{aligned}
& H_{11}=m_{1}+m_{2}+m_{c} \\
& H_{12}=-\left(\frac{1}{2} m_{1} l_{1}+m_{2} L\right)+\frac{1}{2} m_{2} l_{2} \\
& H_{13}=\frac{1}{2} m_{2} l_{2} \\
& H_{21}=-\left(\frac{1}{2} m_{1} l_{1}+m_{2} L\right)+\frac{1}{2} m_{2} l_{2} \\
& H_{22}=\frac{1}{4} m_{1} l_{1}^{2}+m_{2} L^{2}+\frac{1}{4} m_{2} l_{2}^{2}-m_{2} L l_{2}+J_{1}+J_{2} \\
& H_{23}=\frac{1}{4} m_{2} l_{2}^{2}-\frac{1}{2} m_{2} L l_{2}+J_{2} \\
& H_{31}=\frac{1}{2} m_{2} l_{2} \\
& H_{32}=\frac{1}{4} m_{2} l_{2}^{2}-\frac{1}{2} m_{2} L l_{2}+J_{2} \\
& H_{33}=\frac{1}{4} m_{2} l_{2}^{2}+J_{2} \\
& \frac{\partial G}{\partial q}=\left[\begin{array}{ccc}
0 & 0 & 0 \\
0 & -\left(\frac{1}{2} m_{1} l_{1}+m_{2} L\right) g+\frac{1}{2} m_{2} l_{2} g & \frac{1}{2} m_{2} l_{2} g \\
0 & \frac{1}{2} m_{2} l_{2} g & \frac{1}{2} m_{2} l_{2} g
\end{array}\right] \\
& B=\left[\begin{array}{c}
{\left[\begin{array}{c}
0_{3 \times 1} \\
H^{-1}\left[\begin{array}{l}
1 \\
0 \\
0
\end{array}\right]
\end{array}\right]} \\
H^{2}
\end{array}\right]
\end{aligned}
$$

Using the physical system parameters listed in Section III, a Linear Quadratic Regulator (LQR) controller was designed to simultaneously stabilize the IP while keeping the cart at the center of the track. The cart postion/velocity and IP angle/angular velocity are available for feedback. The nominal feedback gains are: 
[-14.1 48.4 -16.8 8.4] with the forward loop gain $K_{T}$ set to 1. Figure 8 shows a root locus plot of the linearized system with varying forward loop gain. The shape of the root locus plot is remarkably similar to that of a unstable launch vehicle with an unstable slosh mode. ${ }^{3}$ The double integrator is associated with the cart dynamics. The IP dynamics is unstable (two real axis poles with one being on the positive real axis). The slosh pendulum dynamics is oscillatory with two poles located near the imaginary axis. For the system shown in Fig. 6, the SP pendulum is at a location that is phase unstable. This is apparent from the angle of departure associated with the slosh loci for which it moves initially into the right half plane. The closed-loop system is conditionally stable as long as $K_{T}$ remains within a certain range. In addition, the presence of damping would shift the slosh dipole location further into the left half plane.

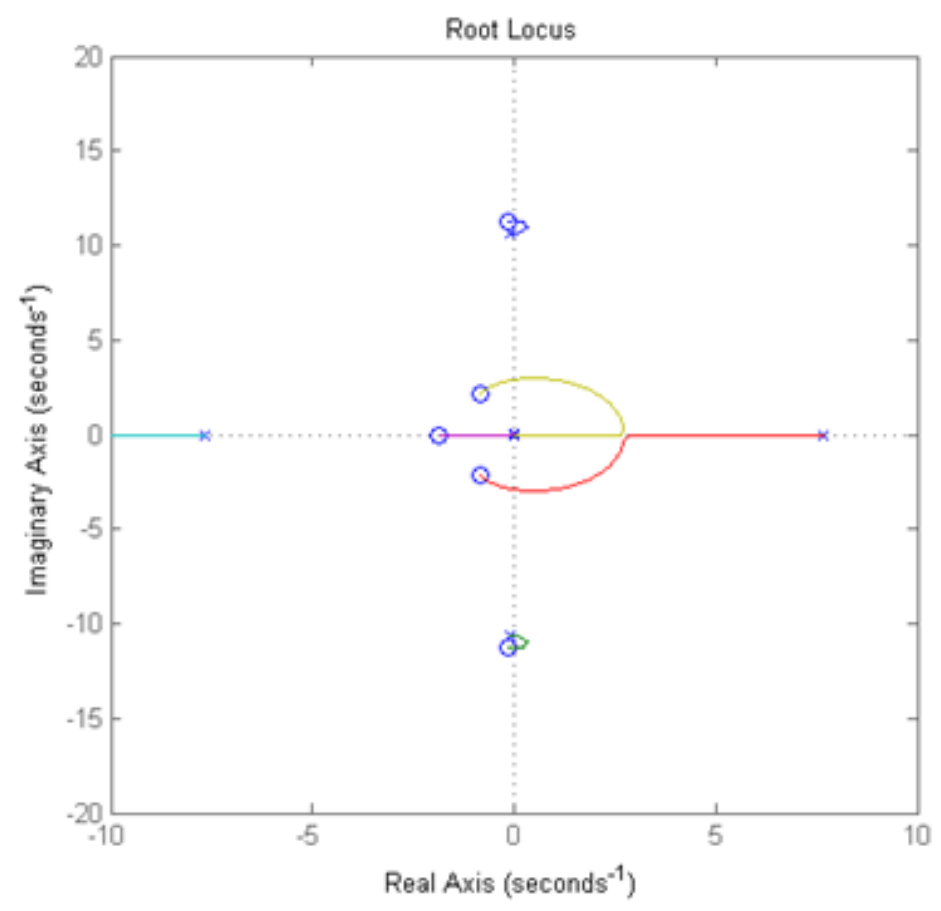

Figure 8. Root Locus of the Linearized Pole-cart system

\section{Experimental Setup}

Figure 9 shows the pole-cart system setup at the LaRC's Dynamic Sytems and Control Branch's (DSCB) Aircraft Controls Research Lab (ACRL). The setup consists of a host computer, a target computer, a data acquisition board, input/output connection block, power unit that drives the motor, and the cart double pendulum hardware. The cart position and the angle of the inverted pendulum are sensed using two quadrature optical encoders. The host computer is used for the design/modeling of the plant and controller. The target computer runs Mathworks XPC Target, which executes the model and controls the hardware in real-time. The motor dynamics can be approximated by Eq. (13), where $F$ is the input force to the cart and $V$ is the commanded voltage.

$$
F=\frac{K_{m} K_{g}}{R_{m} r} V-\frac{1}{R_{m}}\left(\frac{K_{m} K_{g}}{r}\right)^{2} \dot{x}
$$




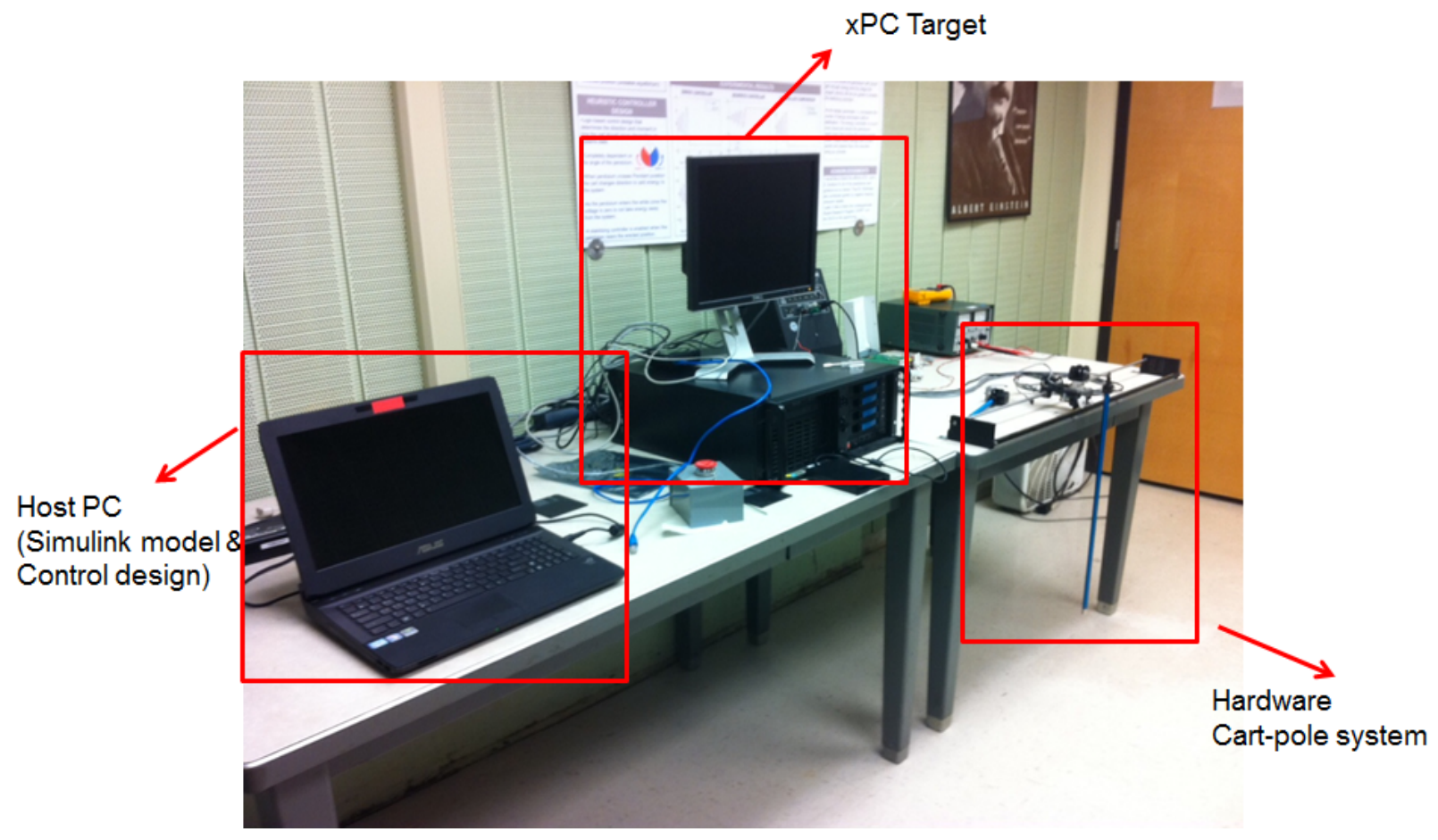

Figure 9. Experimental Setup

Constant parameters include mass of the cart, mass/length of the IP, and mass/length of the slosh pendulum(s). The free parameter is the pivot location of the slosh pendulum with respect to the IP. Table 1 tabulates the value of each parameter.

Table 1. System Parameters

\begin{tabular}{cc}
\hline \hline System Parameters & Values \\
\hline Mass Cart & $0.56 \mathrm{~kg}$ \\
Mass Inverted Pendulum & $0.28 \mathrm{~kg}$ \\
Mass Long Slosh Pendulum & $0.05 \mathrm{~kg}$ \\
Mass Short Slosh Pendulum & $0.025 \mathrm{~kg}$ \\
Length Inverted Pendulum & $0.64 \mathrm{~m}$ \\
Length Long Slosh Pendulum & $0.185 \mathrm{~m}$ \\
Length Short Slosh Pendulum & $0.115 \mathrm{~m}$ \\
Motor armature resistance, $R_{m}$ & $2.6 \Omega$ \\
Back EMF constant, Km & $0.00767 \mathrm{Nm} / \mathrm{A}$ \\
Gearbox ratio, Kg & 3.7 \\
Wheel Radius, $\mathrm{r}$ & $0.0064 \mathrm{~m}$ \\
\hline \hline
\end{tabular}




\section{Results}

In this section, selected results from numerical simulation and experiment are shown. Once again, the intent is to use the pole-cart system to represent the effect of fuel slosh on the closed-loop attitude control of a launch vehicle. All parameters were fixed except for $\mathrm{L}$, distance between the pivot points $\mathrm{P}$ and $\mathrm{Q}$ shown in Fig. 10. Depending on the value of L, the SP could be either phase stable or unstable relative to direct control of the IP. This concept is analagous to the slosh danger zone on a launch vehicle as described in Section I, although a direct one-to-one link does not exist. If the SP is phase unstable, the amount of damping becomes critical in the stability of the system. On a launch vehicle, this would be equivalent to adding baffles or increase the roughness of the slosh tank wall. If the SP is phase stable, the control system actively seeks to reduce the energy of the SP. Only two cases are presented in this paper: 1) L $=0.42 \mathrm{~m}$ (SP phase unstable), 2) $\mathrm{L}=0.00 \mathrm{~m}$ (SP phase stable). In addition, slosh pendulums of different length were used in the study. The length and mass of the slosh pendulum directly affects its natural frequency and damping. Euler's method was used as the integration scheme in the numerical simulation with a step size of 1.0e-4 seconds. Additionally, first order motor dynamics were included.

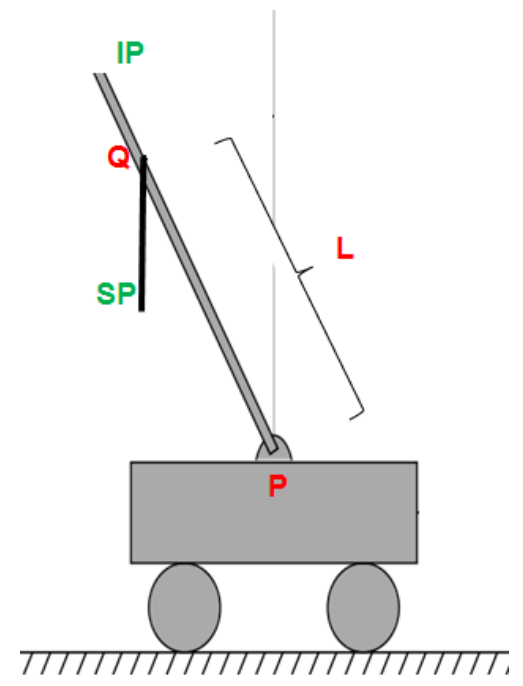

Figure 10. Experimental Setup

\section{A. Experimental Estimation of Damping}

Damping values of the two slosh pendulums were extracted from calibration experiments by releasing the pendulums at non-zero initial angles. For small angles $(<15 \mathrm{deg})$, the response of the system to initial conditions is fairly linear, hence a second order linear model was fit to the experimental data. Figure 11 shows the response of the short slosh pendulum to a non-zero initial condition. The second order fit yielded a damping $(\zeta)$ value of 0.035 . Figure 12 shows the response of the long slosh pendulum to a non-zero initial condition with a second order model $(\zeta=0.02)$. These damping values were subsequently used in the numerical simulations. 


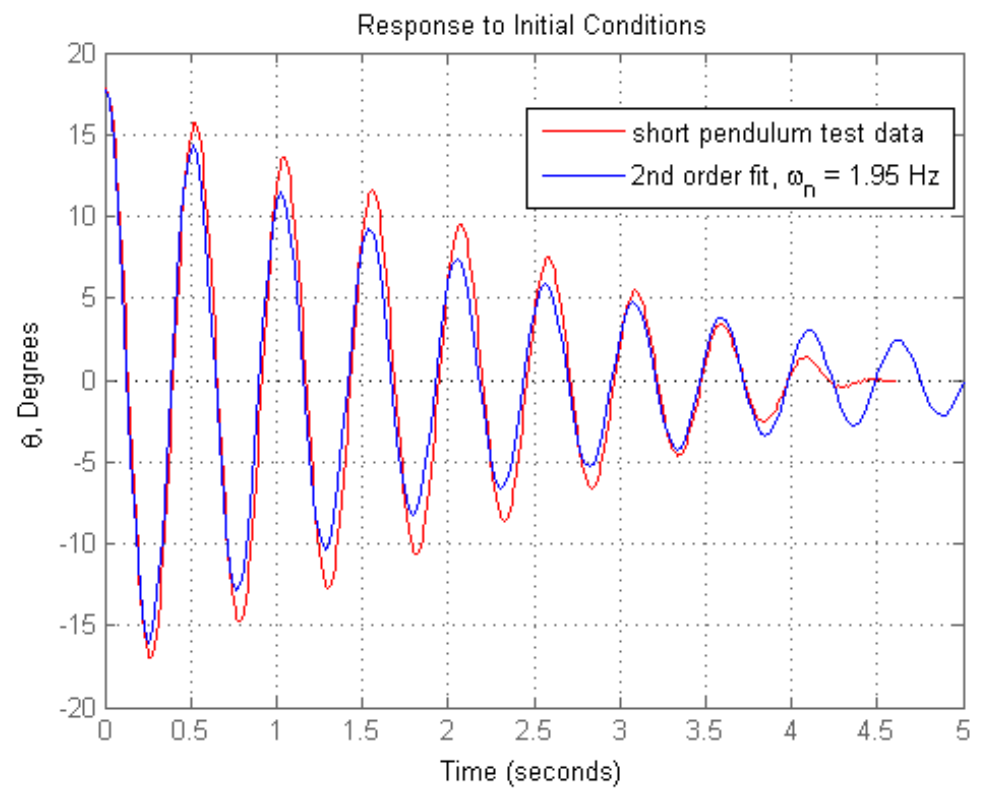

Figure 11. Damping estimation: short slosh pendulum

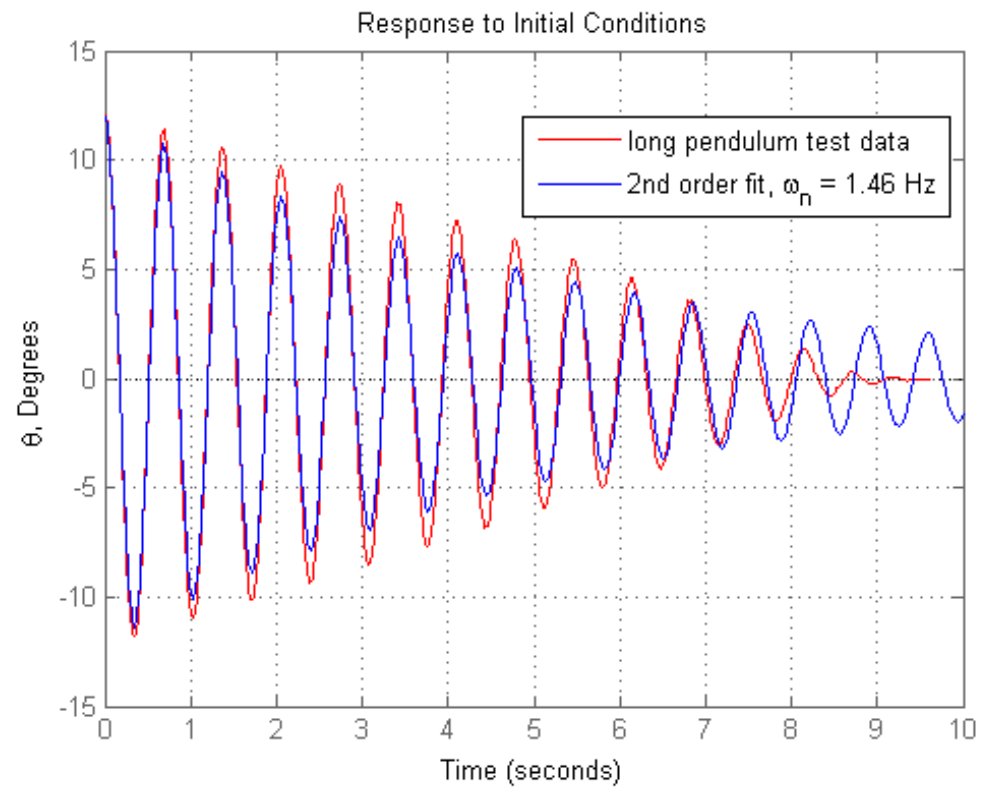

Figure 12. Damping estimation: long slosh pendulum

\section{B. Experimental Results}

Selective experimental results are shown in this subsection. For each run, the system started out at the equilibrium positions until a force was applied to excite the slosh pendulum. Figure 13 illustrates the time histories of key system parameters for the long slosh pendulum at $\mathrm{L}=0.42 \mathrm{~m}$. Subplots 1 and 2 are the phase portraits of the inverted pendulum and the cart (postion vs. velocity). Subplot 3 is the voltage command to the motor and subplot 4 shows the time history of the slosh pendulum angle. The divergent behavior of 
$\theta_{2}$ shows that the slosh mode is unstable. It hits the hardware limits of $(+/-80 \mathrm{deg})$ in roughly 15 seconds and the instability is also apparent in the control time history as it reaches the $+/-4$ volts saturation limits simultaneously.
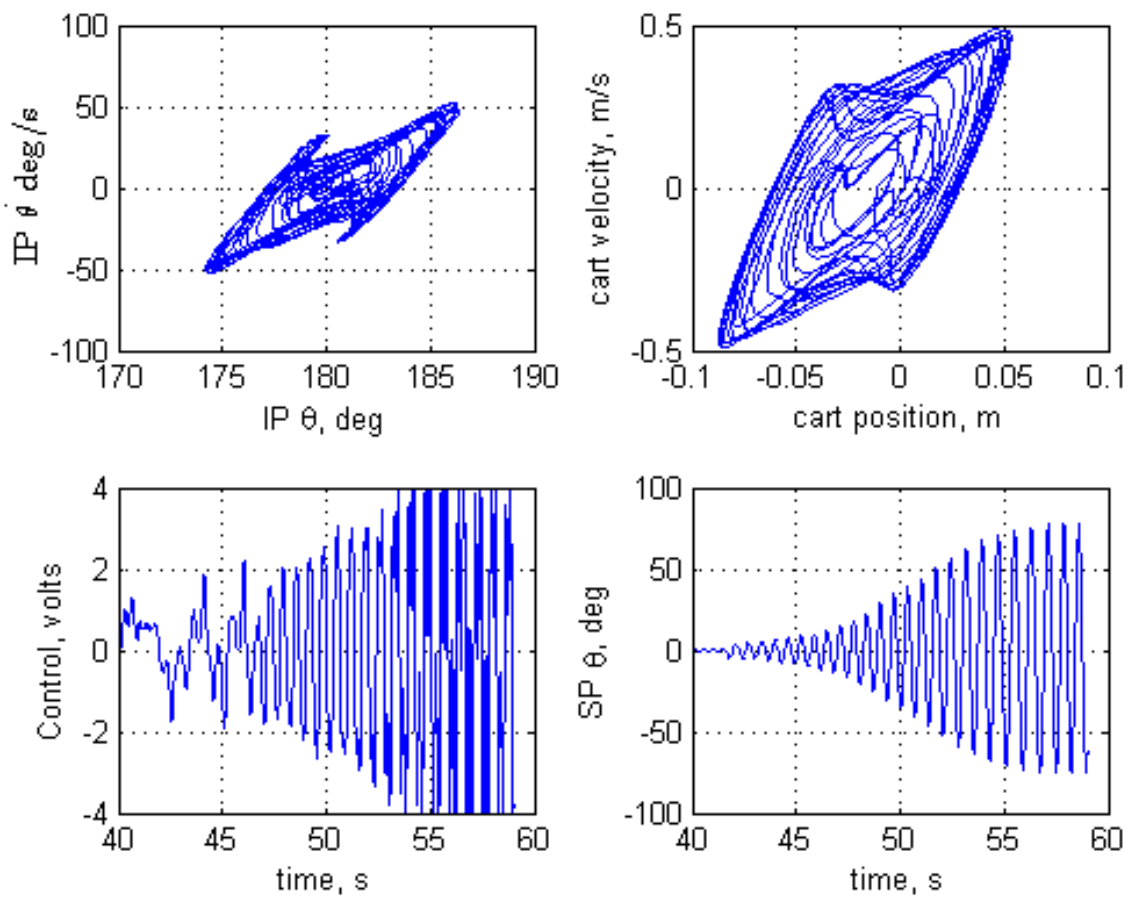

Figure 13. Experimental Results: Long Slosh Pendulum, L $=0.42 \mathrm{~m}$

Figure 14 is the experimental results for the short slosh pendulum at $\mathrm{L}=0.42 \mathrm{~m}$. Again the system initially started from the equilibrium position until an impulse was applied to excite the slosh pendulum at $\mathrm{T}=32$ seconds. Unlike with the long pendulum at $\mathrm{L}=0.42 \mathrm{~m}$, the slosh pendulum angle damps out after roughly 12 seconds. Linear stability analysis suggests that the slosh mode is phase unstable and the amount of damping in the system is able to stabilize the slosh dipole by preventing the frequency response from encircling the critical point. Figure 15 shows the Nichols chart of the phase unstable slosh mode represented by Figure 14. At $\mathrm{T}=45$ seconds, a larger pulse was applied to the slosh pendulum. This time the slosh amplitude grew and reaches a limit cycle. Unlike the long slosh pendulum case, the control never reaches the saturation limit and the slosh pendulum never hit the hardware limits. The larger impulse caused the system to exit the linear region and nonlinear dynamics begins to dominate. For long slosh pendulum, damping was not sufficient to prevent the system from instability whether the initial impulse was large or small. 

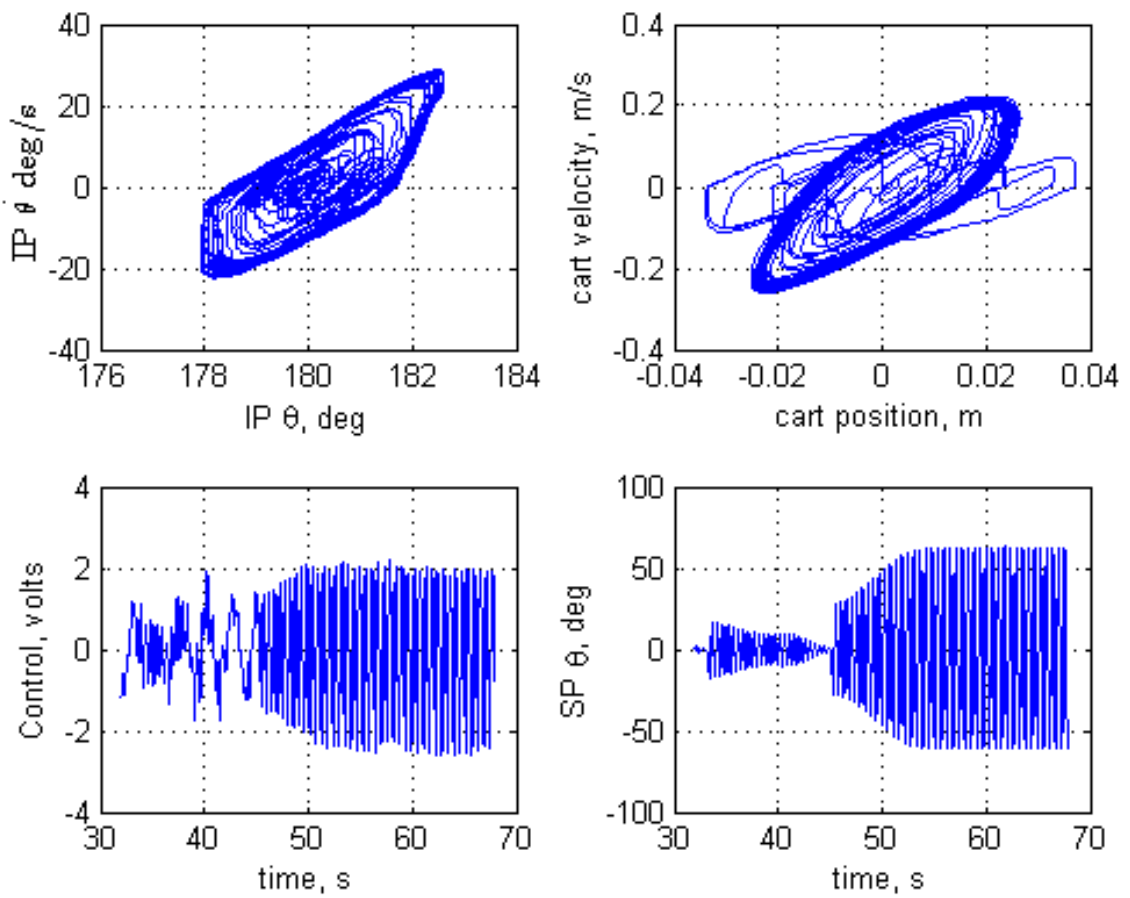

Figure 14. Experimental Results: Short Slosh Pendulum, L $=0.42 \mathrm{~m}$

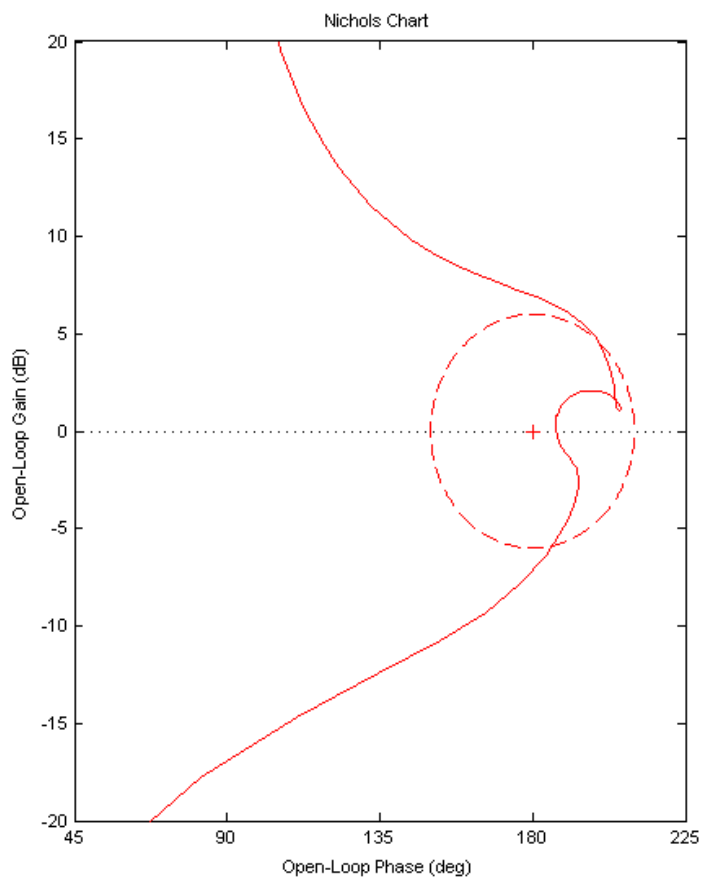

Figure 15. Nichols chart: Short Slosh Pendulum, $\mathrm{L}=0.42 \mathrm{~m}$

Figure 16 shows the experimental results for the long slosh pendulum at $\mathrm{L}=0 \mathrm{~m}$. Linear analysis indicates 
that for this configuration the slosh dipole is phase stable. At $\mathrm{T}=12$ seconds, a small pulse was applied to the slosh pendulum, the amplitude decayed as expected. At $\mathrm{T}=28$ seconds, a larger pulse was applied and again the system came to rest after some time. The short slosh pendulum exhibited the same behavior with a faster decay rate attributed to the higher damping.
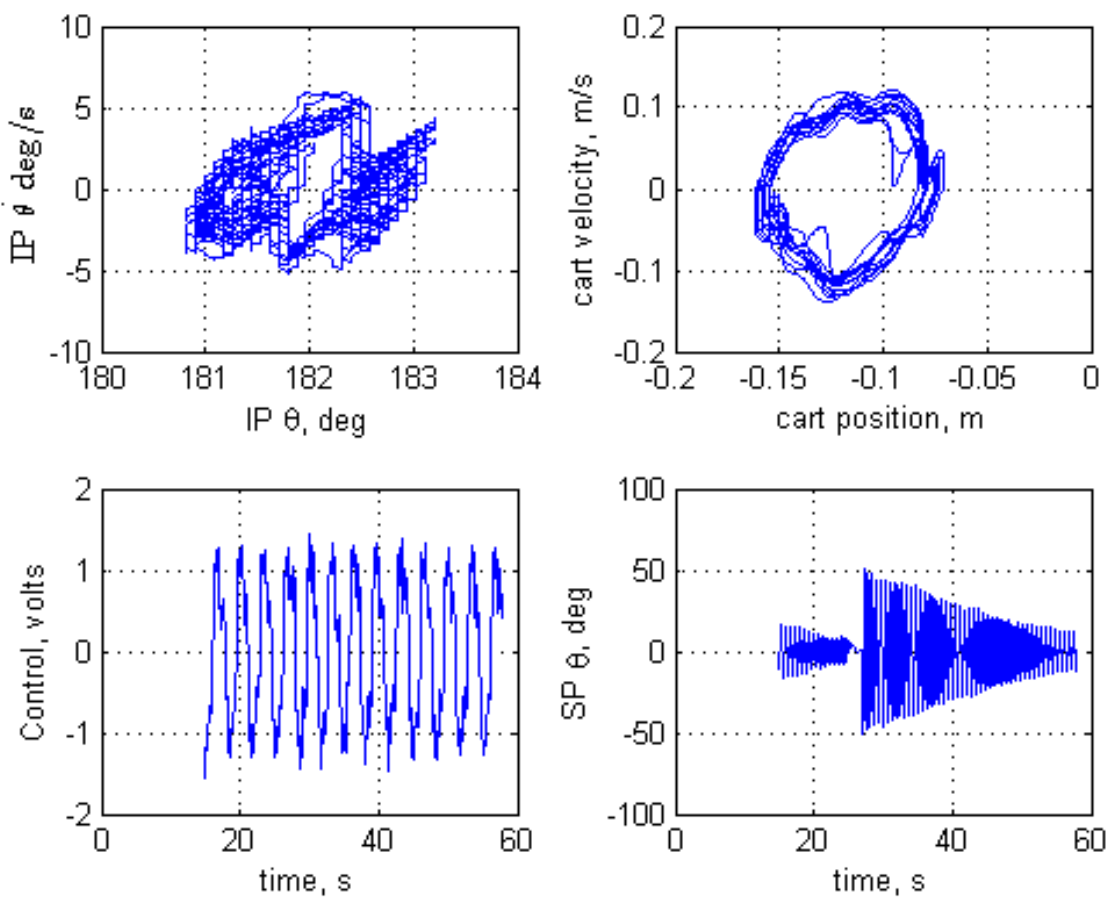

Figure 16. Experimental Results: Long Slosh Pendulum, L $=0.00 \mathrm{~m}$

\section{Numerical Results}

Corresponding numerical simulations and linear stability results are shown in this section to provide analytical insight to the experimental results. With linear analysis it is easy to visualize whether the slosh mode is naturally stable with respect rigid body control. However at this point, numerical simulation results do not match the experimental results exactly due to unmodeled dynamics and nonlinearities. The estimated linear damping profile was used in the simulation even though in reality the damping profile is a nonlinear function of the pendulum angles. Figures 17 and 18 are the Nichols chart and simulated time domain results (nonlinar EOM + linear damping) for the long slosh pendulum at $\mathrm{L}=0.42 \mathrm{~m}$. The slosh dipole is phase unstable and unlike for the short pendulum (Fig. 15) there is not adequate damping in the system to prevent the frequency response from closely approaching the critical point. Nevertheless, the system is still stable from a linear standpoint and this is apparent in the simulated time domain results. Figure 19 shows if the estimated 2 percent linear damping value is reduced then simulation matches the experimental results as shown in Figure 13. In this case, similar to the experiment, both the control output and slosh pendulum angle both reaches the saturation limits within approximately 15 seconds. 


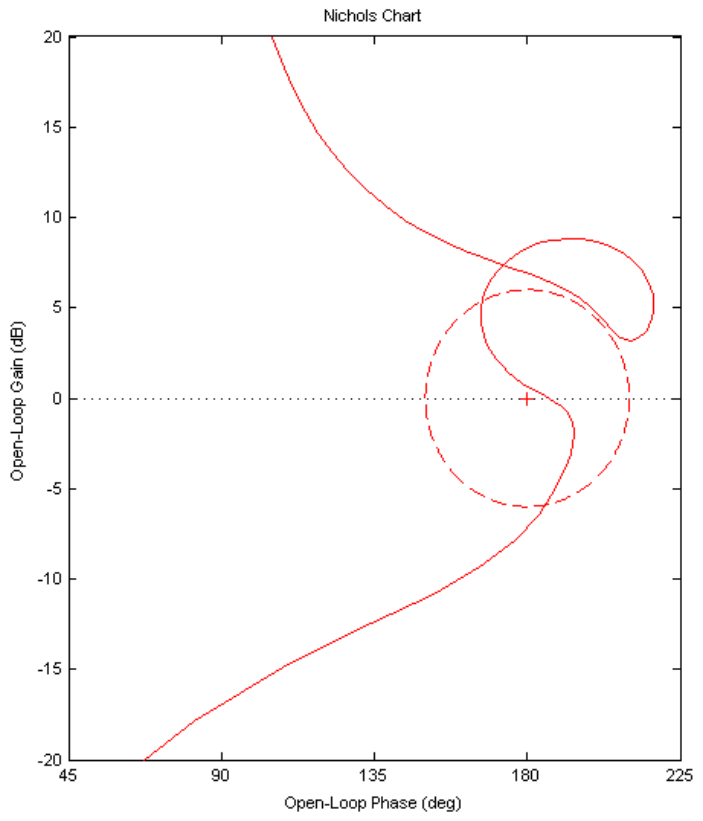

Figure 17. Nichols chart: Long Slosh Pendulum, L $=0.42 \mathrm{~m}$
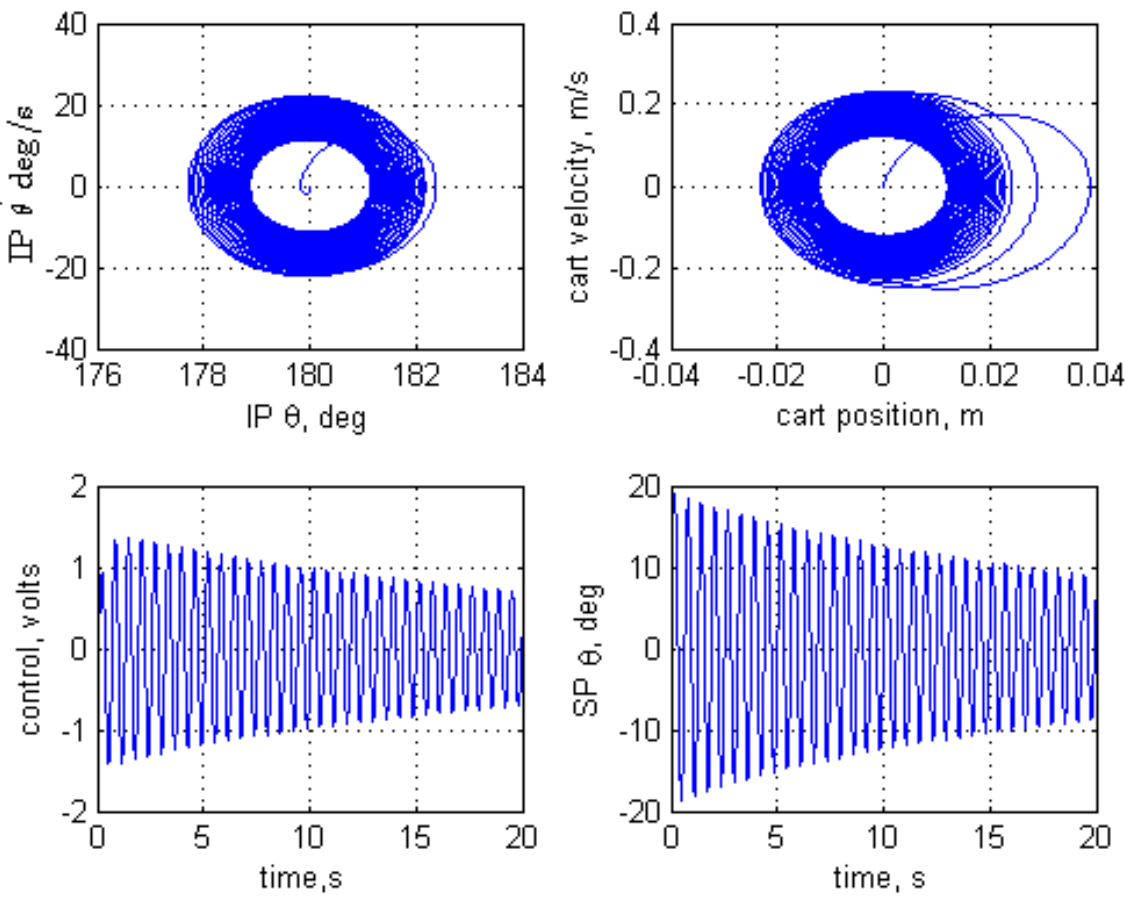

Figure 18. Numerical Simulation: Long Slosh Pendulum, L $=0.42 \mathrm{~m}$ 

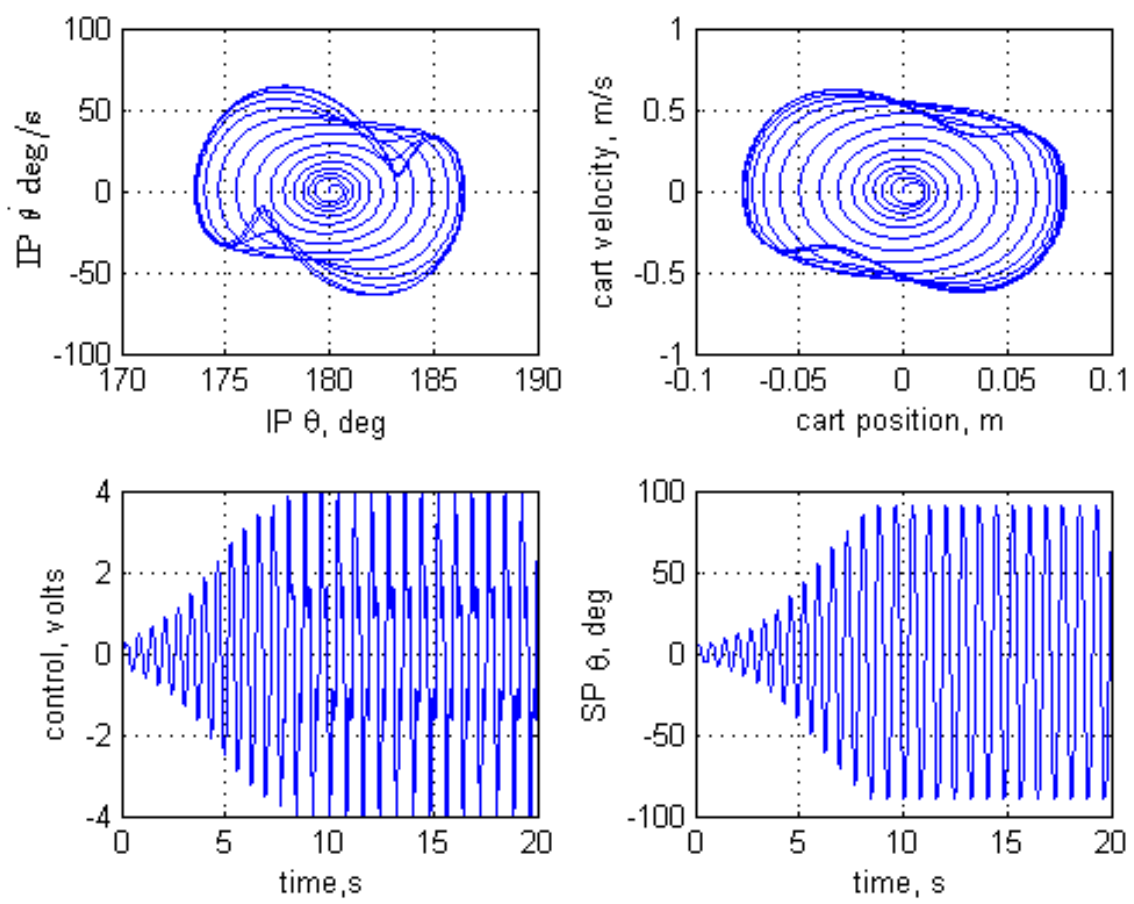

Figure 19. Numerical Simulation: Long Slosh Pendulum, $\mathrm{L}=0.42 \mathrm{~m}$, reduced damping

Figures 20 and 21 are the Nichols chart and simulated time domain results for the long slosh pendulum at $\mathrm{L}=0.00 \mathrm{~m}$. The slosh loop in the frequency response is now phase stable with respect to rigid body control as it circles away from the circle point. Even if damping is absent, the control would seek to actively take away energy from the slosh mass. This is consistent with experimental results shown in Fig. 16. The short slosh pendulum has a similar frequency response, except the slosh loop is smaller due to additional damping. 


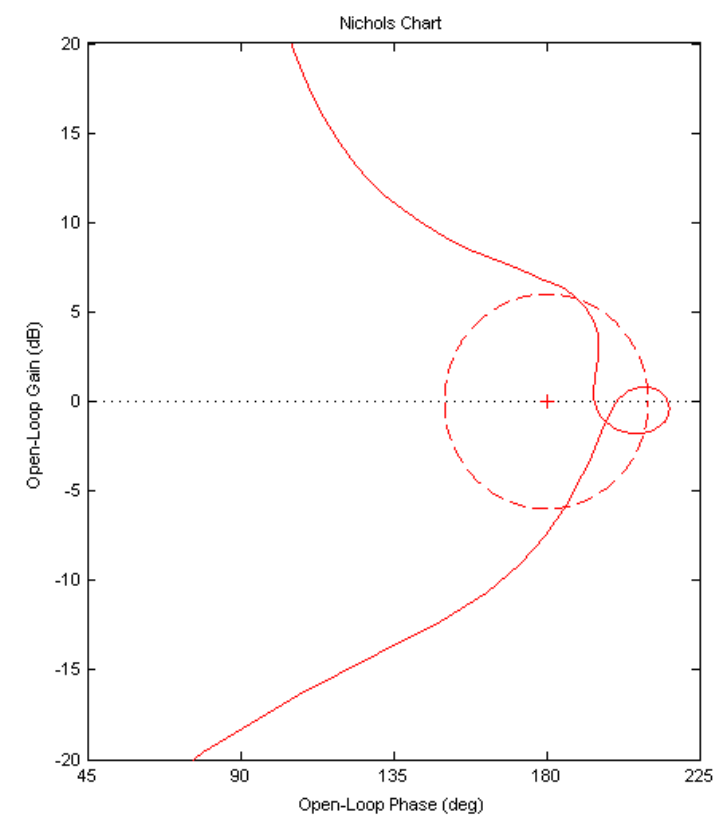

Figure 20. Nichols chart: Long Slosh Pendulum, $\mathrm{L}=0.00 \mathrm{~m}$
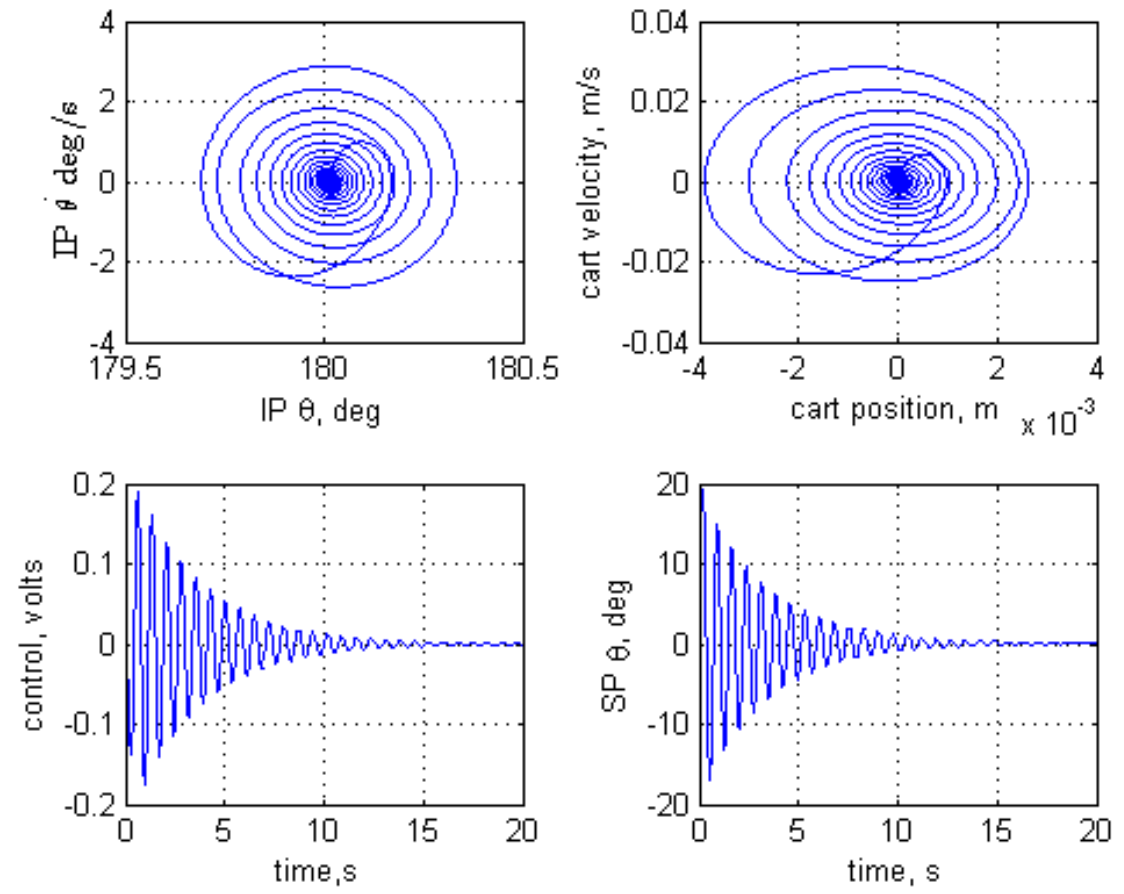

Figure 21. Numerical Simulation: Long Slosh Pendulum, $L=0.00 \mathrm{~m}$

As described in the introduction, the center of percussion is defined as a point on the body where the translational motion perfectly cancels out the rotational motion at a given instance in time. Substituting the system parameters into Eq. (1) suggests that the center of percussion of the pole-cart system is at $\mathrm{L}=$ 
$0.037 \mathrm{~m}$ as measured from the pivot point $\mathrm{P}$. Whereas for a launch vehicle, the CP is measured relative to the center of mass. Linear analysis suggests that at roughly $\mathrm{L}=0.04 \mathrm{~m}$, the phase lead associated with the slosh dipole on the pole-cart system disappears and the frequency response begins to appear in the unfavorable direction. Figure 22 shows the Nichols chart for the long slosh pendulum at $\mathrm{L}=0.05 \mathrm{~m}$.

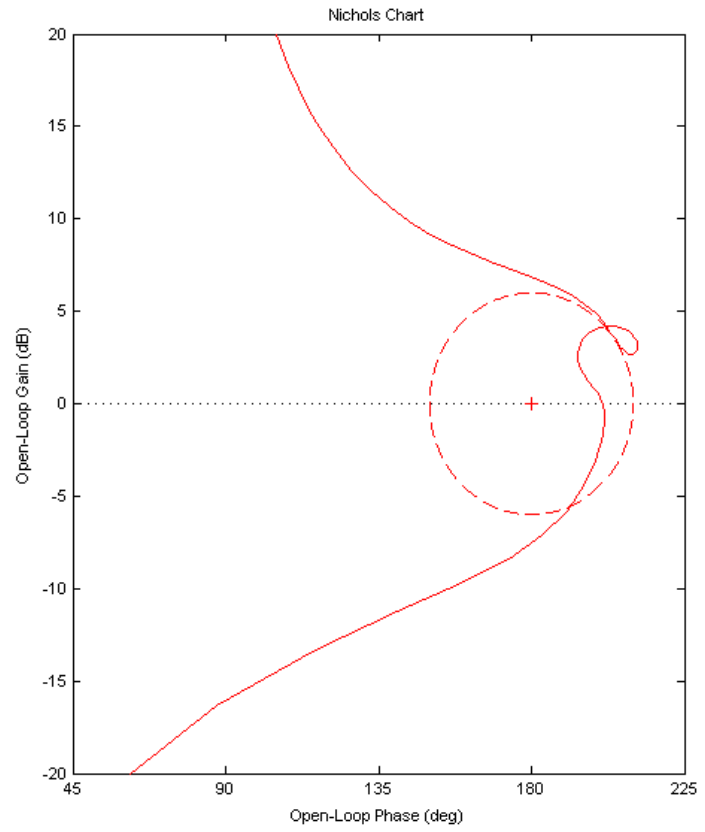

Figure 22. Nichols chart: Long Slosh Pendulum, $\mathrm{L}=0.05 \mathrm{~m}$

\section{Conclusion}

Propellant slosh dynamics has a pronounced influence on the closed loop stability of a launch vehicle. The pole-cart system provides an excellent test platform for launch vehicle dynamics and control applications. In this paper, the effect of a single slosh dipole on the attitude control of a launch vehicle was demonstrated using the double pole-cart system. Experimental mismatches with simulation at certain conditions point out the importance in understanding unmodeled dynamics and system nonlinearities. Nevertheless, analytical results are valuable in gain insight to the fundamental system dynamics. For both systems (launch vehicle or pole-cart), depending on the location of the slosh mass relative to the rigid body, it could be either phase stable or unstable. In the case of poor phasing, sufficient damping is required to passively stabilize the system. In the case of launch vehicles, this means additional baffles or increase tank wall roughess. For the pole-cart system, this means an increase in the friction coefficient at the slosh pendulum pivot point. This fact was demonstrated on the pole-cart platform in both experimental and simulation settings. In addition, the experiments indicated that increasing the slosh mass (longer SP) or decreasing the slosh natural frequency (longer SP) degrades the stability of the rigid body. This observation is consistent with analysis performed by Bauer. For future work, the simulation will be improved to better represent the actual hardware. In addition, multiple slosh pendulums may be attached to the inverted pendulum to mimic the effect of mutual interference between slosh tanks. 


\section{References}

${ }^{1}$ Bauer, H., "Fluid Oscillation in the Containers of a Space Vehicle and Their Influence Upon Stability," Tech. Rep. TR R-187, NASA Technical Report, 1963.

${ }^{2}$ SpaceX, "Falcon Demo Flight 2. Flight Review Update," Tech. rep., SpaceX, July 2007.

${ }^{3}$ Greensite, A., "Analysis and Design of Space Vehicle Flight Control Systems. Volume VII. Attitude Control During Launch," Tech. Rep. CR-826, NASA Contractor Report, July 1967.

${ }^{4}$ Orr. J, J. M., "Space Space Implementation of Linear Pertubation Dynamic Equations," AIAA, 2009.

${ }^{5}$ Bauer, H., "Stability Boundaries of Liquid-Propelled Space Vehicles with Sloshing," AIAA Journal, Vol. 1, No. 7, July. 1963.

${ }^{6}$ Beer and Johnston, Statics and Dynamics, McGraw Hill, six ed., 1997.

${ }^{7}$ Buonocunto, P. and Corucci, F., "Real-Time PID control of an Inverted Pendulum," MS Thesis, University of Pisa, Italy.

${ }^{8}$ Krishnan, R., "On Stabilization of Cart-Inverted Pendulum System, An Experimental Study," MS Thesis, National Institute of Technology, Rourkela, India.

${ }^{9}$ Tedrake, R., "Underactuated Robotics Class Notes," MIT.

${ }^{10}$ Spong, M., "Underactuated Mechanical System," Tech. rep., University of Illinois of Urbana Champaign, 1997.

${ }^{11}$ Shaub and Junkins, Analytical Mechanics of Space Systems, AIAA, 2003. 